\title{
Brief Introduction to Ceramic Material Application in Modern Bamboo Furniture Design
}

\author{
Jiamin Huang ${ }^{1, a}$, Wenyin Dong ${ }^{1,2, b}$,Shuhang Zhang ${ }^{1, c}$ \\ ${ }^{1}$ Forestry College, Sichuan Agricultural University, Chengdu, China \\ ${ }^{2}$ Central South University of Forestry and Technology, Changsha, China \\ a474752253@qq.com, bdoreen0656@126.com, ${ }^{c 815942971 @ q q . c o m ~}$
}

Keywords: ceramic material; bamboo furniture; ornaments; design

Abstract. Based on the application of ceramic furniture and material in traditional furniture design, analyze the probability of ceramic material application in modern bamboo furniture design and further search the methods of such application. It can enrich the design content and pattern of manifestation of modern bamboo furniture which provides theoretical reference for ecological modern bamboo furniture design mainly ornamented by ceramic material.

\section{Introduction}

Ceramics and bamboo are considered as Chinese traditional materials. Ceramic exquisite texture conveys the sense of nobleness, though it cannot be designed in various shapes because of its fragility. Bamboo is endowed with the sense of dignity that fresh and natural texture, however it is prone to mustiness and worm corrosion which cannot meet the optimal material requirements. These respective defects make it impossible to achieve the optimum in the course of processing and application. The combination between ceramics and bamboo not only inherits Chinese history and culture, but also complements each other to bring about the design diversity. Adopting the simple style of modern furniture, the combination will lead the brand new direction of modern furniture design.

\section{Ceramic Material Application in Furniture Design}

Ceramics, the representative of Chinese traditional creation forms, also becomes the design material of traditional furniture. Through development and accumulation of several thousand years, Chinese ceramics has formed various artistic features. Ceramics is not only designed for practical devices but as artistic ornaments. In this senseceramics is not only considered as material products but as spiritual and cultural ones ${ }^{[1]}$. Often used indoors, furniture is highly required in material. With high strength, high hardness, anti-corrosion and high temperature resistance, ceramics has become a kind of superior material of furniture design. In all ages, ceramics is well applied in furniture design.

Ceramic Material in Traditional Furniture.

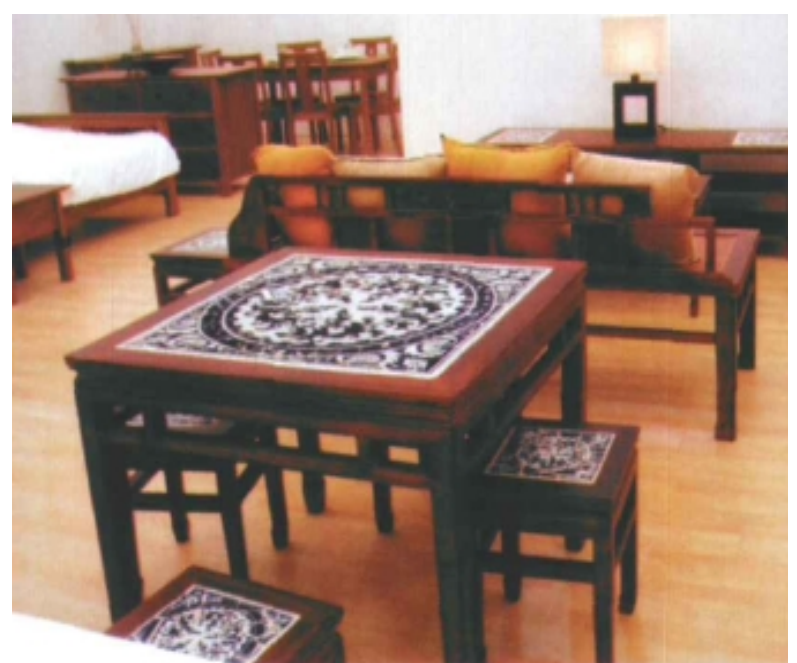

Fig. 1 Traditional ceramic tables and stools

In Chinese ancient times, ceramics and timber were employed to make furniture. According to historical records, the earliest ceramic furniture came up in the Sui and Tang dynasties, and prevailed in the Ming and Qing dynasties ${ }^{[2]}$. Ceramic material application in furniture can be divided into two types. One is a large area of thick porcelain panels with colorful paintings as a substitution for part of the furniture, such as ceramic tables, ceramic stools and so on. As shown in the first picture, the 
product partly inset with blue and white porcelain pieces presents the feelings of simplicity and elegance. Such products enjoy the popularity because it not only cater to the tendency of modern simple design, but also inherit with traditional artistic tastes ${ }^{[3]}$. The other is a small area of ceramics as accessories, embedded in furniture or cabinet doors and screens. The second picture is the Huanghuali wood officer' $s$ hat armchair with four flat protruding ends, embedded with blue and white ceramic panels of dragon and phoenix paintings, which is collected in Beijing Guanfu Museum. The wooden structure decorated with blue and white ceramic panels of dragon and phoenix paintings makes itself pure, fresh and free from vulgarity, especially full of charm. The combination brings out the best state.

Ceramic Material in Modern Furniture. Following the idea of modern design, modern furniture is mainly made of new materials and it is also variously designed. The utilization of new materials or different materials in standardized production mode adds to a sense of modern times and elaborate design, which brings convenience and visual aesthetic feelings to our life. The combination between ceramics and other materials just like wood, glass, plastic, metal and bamboo gives rise to the comparison and the complement. Such design not only makes the users feel the sense of relaxation and nature

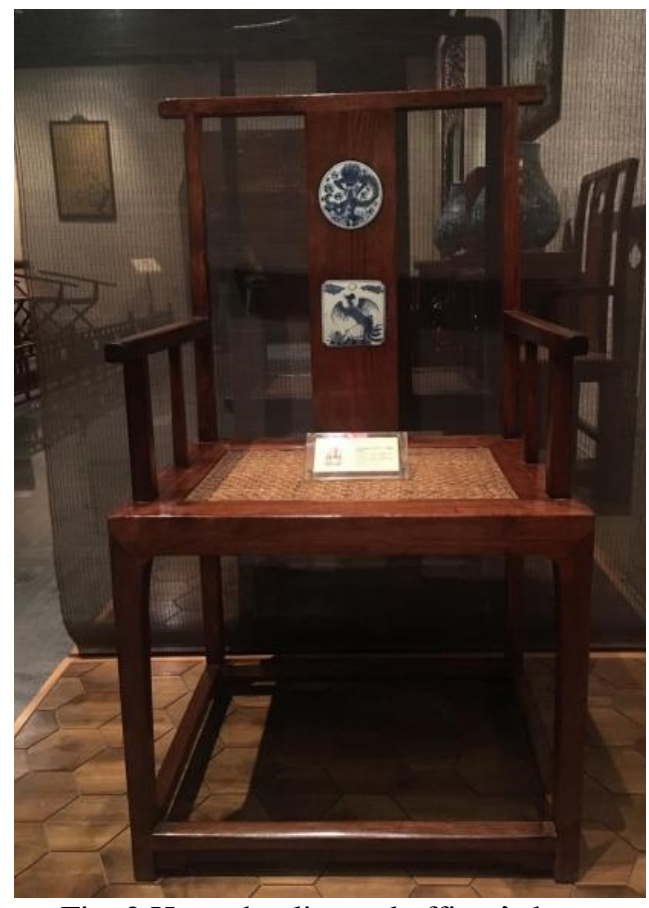

Fig. 2 Huanghuali wood officer's hat armchair with four flat protruding ends, embedded with blue and white ceramic panels of dragon and phoenix paintings which brought by modern simple design, but also arouses the resonance towards the artistic culture of traditional decoration ${ }^{[3]}$. These actions can provide certain reference for the combination design between ceramics and bamboo.

\section{Analysis of the Ceramic Material Application in Bamboo Furniture Design}

Feasibility Analysis of the Ceramic Material Application in Bamboo Furniture Design. Ceramics and bamboo have in common both in the perspective of ideological cultural and modern industrial production. However, the material limitation still exists. Therefore, analyze the differences between them, ingeniously find the common elements, exclude the defects and finally make the combination more natural in a brand new design idea of applying ceramic elements to modern bamboo design decorations. In this way, we can design high-quality modern ceramic bamboo furniture.

Similarity Between Ceramics and Bamboo.

Humanity Characters - the Continuation of Chinese Traditional Culture and Thoughts. From the respective of cultural carrier, the aesthetic thoughts, performance skills and mature development of ceramic materials all reflect the Chinese traditional culture. The word of China has been closely connected with the word of china that stands for Chinese porcelain, with the wide spread of Chines porcelains in Britain and other European countries ${ }^{[4]}$. The ceramic characteristics of soft quality, varied colors, rich texture and smooth tactility represents the abundant Chinese cultural heritage. As explained in Chinese traditional ideology, the natural characters of smooth tactility, fresh fragrance and simple texture symbolize the noble quality and quiet attitude of Chinese national spirits. In terms of humanity characters, the symbol meanings of ceramics and bamboo consist with public aesthetic taste. Moreover, they contain intense eastern aesthetic connotations and meet the diversified demands of 'poetic residence' that modern design is pursuing. The combination between traditional culture and modern arts is not only material continuation of Chinese culture but also cultural heritage of traditional spirits. 
Material Character - Environmental-friendly Material. In terms of material production, as both traditional and new materials, some problems can be solved with the support of modern scientific technology despite the respective defects. The fragility problem of ceramic materials can be solved by the Nanotechnology while the hardness and worm corrosion problem can also be solved by the industrial technology of integrated materials. The artificial ceramics is made from soil under the high temperature. Ceramics can be widely developed and produced in the long period just because of modern crafts in the operable production process. On account of stable character, ceramics is considered as a sustainable and environmental-friendly furniture material. As the distribution center of bamboo, China is the country in the world that enjoys the most abundant bamboo resources and cultivates the bamboo earliest. Bamboo is ideal material of furniture and floor for its good physical characters of straight texture, elegant colors, hard wood and other good characters of hard-broad leaf wood. With an excellent thin-wall and hollow tube structure, it can be made into curved furniture. Compared with other materials, ceramics and bamboo own the unique charm and can be applied to modern furniture design as environmental-friendly material.

Differences Between Ceramics and Bamboo.

Just for the differences, they can complement each other and bring about the best. Compared with bamboo, ceramics (silicate materials) owns the characteristics of high hardness, wear-resisting property, resistance to acid and alkali, resistance to heat and cold, and so on. However, they are extremely fragile. Ceramic furniture could meet with problems of functional defects and technical difficulty. For example, ceramics is hard to cut and drill just for physical characteristics, which increase the difficulty of combining ceramics with wood, metal and other material. In addition, its low resistance to shock, namely its fragility, restricts the application range ${ }^{[1]}$.On the contrary, bamboo has the strong mechanical characteristics of resistance to bending, crushing and dragging and could become soft under the high temperature. The material can be shaped into different arcs under external force and falls into a fixed pattern by rapid cooling. But it also remains prone to mustiness and worm corrosion. The combination between ceramics and bamboo can solve many problems in the process of production and utilization.

\section{Ceramic Material Application Techniques in Bamboo Furniture Design.}

Embedded Parts. The integrally embedded techniques are traditional skills that combining ceramics and furniture as superficial and partial ornaments. In traditional furniture, the techniques of embedded ceramic materials are quite mature both on the function of utility and aesthetic. On this basis, considering the hollow tube feature of bamboo, modern bamboo furniture design should utilize the innovative technology to make the combination natural.

Embedded Knitting. Based on the pliability of bamboo, combine the knitting crafts between ceramic pieces and bamboo pieces and further embed ceramic pieces into bamboo knitting products. The combination between well-organized knitting and beautiful ceramic patterns gives rise to a new visual effect.

Decorated Elements. In traditional furniture, ceramic products are decorated with some accessories, such as handles, bolts, blades, truckles and so on ${ }^{[3]}$. In modern furniture design, enlarge the decorated elements, such as four corners or corner lines of bamboo desks, and furniture feet that can prevent from water and moisture.

\section{Conclusion}

Contemporary furniture designers should employ local culture and meanwhile must consider scientific achievements on new materials and apply them to the design process ${ }^{[1]}$. The combination between traditional ceramics and bamboo furniture design is not only a kind of innovative application in material but also inherits traditional ceramic culture. Such combination is a high-level complex process: on one hand, it needs deep understanding of ceramic and bamboo culture; on the other hand, designers need make rational innovations on the basis of fully considering the respective superiority of two kinds of material. 


\section{Acknowledgements}

This work was financially supported by the major project of humanities and social sciences of education department of Sichuan province (16SA0006), the project of Undergraduate Scientific Research Interest of Sichuan Agricultural University, the research program of Allied Social Science Association of Sichuan Agricultural University (2013YB01).

\section{References}

[1] Q.F.Zhang. The primary exploration of modern ceramic furniture design [D].China Academy of Art.2011.

[2] J.J.Wu. The application of ceramic in furniture design[J]. Window of Knowledge (Teacher edition).2013,03:67.

[3] X.Y.Zhou.X.D.Dai. Ceramic Material Application in modern Furniture ornaments [J]. Furniture and Interior Dcoration.2008,06:56-57.

[4] S.H.Wu. Brief Introduction to the cultural connotation and artistry of ancient ceramics [J]. Ceramic Research.2014,04:92-93. 